11

\title{
Поверхностный импеданс сильно индуктивной двухслойной среды
}

\author{
(С В.К. Балханов, Ю.Б. Башкуев, Л.Х. Ангархаева
}

Институт фризического материаловедения СО РАН, 670047 Улан-Удэ, Россия,

e-mail: ballar@yandex.ru

(Поступило в Редакцию 17 мая 2017 г.)

В зимнее время на Северном Морском пути и на соленых озерах Евразии радиотрассы можно моделировать двухслойной средой, где первая среда (лед) является диэлектрическим слоем, а вторая (море, соленая вода) в СДВ-ДВ-СВ и КВ диапазонах радиоволн является подстилающей проводящей средой. Рассматриваемая двухслойная среда с реальными электрофизическими и геометрическими параметрами будет иметь сильно индуктивный поверхностный импеданс. Большой контраст электрофизических свойств слоев льда и воды позволяет выделить в формулах для расчетов поверхностного импеданса малые величины, благодаря чему удается получить конечные аналитические выражения для приведенного поверхностного импеданса (далее импеданс) в различных приближениях по малым величинам. Установлено, что для рассматриваемой среды фаза импеданса лежит в пределах $-45.1^{\circ}--89.9^{\circ}$, что как раз и является критерием сильно индуктивной поверхности.

DOI: $10.21883 /$ JTF.2018.03.45606.2338

\section{Введение}

Мы рассматриваем распространение электромагнитной волны вдоль двухслойной среды „свободное пространство-диэлектрический слой-проводящая подстилающая среда“". Свободное пространство будем маркировать индексом 0. Первый диэлектрический слой маркируется индексом 1 , имеет толщину $h$ и описывается относительной диэлектрической проницаемостью $\varepsilon_{1}$ и удельным сопротивлением $\rho_{1}$. Вторая среда маркируется индексом 2, неограничена по глубине и описывается удельным электрическим сопротивлением $\rho_{2}$ и диэлектрической проницаемостью $\varepsilon_{2}$. Рассматриваемая двухслойная среда является хорошей моделью сильно индуктивной поверхности, служащей радиотрассой для распространяющейся вдоль нее электромагнитной волны. Нашей задачей будет определение аналитического выражения приведенного поверхностного импеданса $\delta$ (далее импеданс) для рассматриваемой сильно индуктивной радиотрассы. Всегда лучше иметь дело с аналитическим выражением. Актуальность задачи связана с тем, что знание импеданса подстилающей среды достаточно для определения всех характеристик радиотрассы и характеристик электромагнитного поля в месте приема радиосигнала. Проверка устанавливаемых ниже формул будет проводиться предельным переходом к однородной проводящей среде с известным импедансом. Установленные аналитические формулы для импеданса будут также сравнены с численным расчетом.

\section{Волновое число}

В каждом однородном слое квадрат волнового числа $k^{2}$ дается следующим известным выражением [1-3]:

$$
k^{2}=\frac{\omega^{2}}{c^{2}}\left(\varepsilon+\frac{i}{\varepsilon_{0} \omega \rho}\right) .
$$

Здесь $\omega-$ круговая частота, $c-$ скорость света, $\varepsilon_{0}$ - диэлектрическая постоянная вакуума. В свободном пространстве $\varepsilon=1$ и $\rho \rightarrow \infty$, тогда квадрат волнового числа

$$
k_{0}^{2}=\frac{\omega^{2}}{c^{2}} .
$$

Для озера Сульфат на юге Сибири первым слоем является лед с диэлектрической проницаемостью $\varepsilon_{1}=3.4$, в марте-апреле месяце имеющий толщину $h=1 \mathrm{~m}$ [4]. Удельное сопротивление льда на соленой воде $\rho_{1}=10^{4} \Omega \mathrm{m}$. В диапазоне частот $100 \mathrm{kHz}-10 \mathrm{MHz}$ величина $1 / \varepsilon_{0} \omega \rho_{1}=18-0.18$. Она одного порядка с величиной $\varepsilon_{1}=3.4$. Поэтому для первого слоя необходимо полагать

$$
k_{1}^{2}=\frac{\omega^{2}}{c^{2}}\left(\varepsilon_{1}+\frac{i}{\varepsilon_{0} \omega \rho_{1}}\right) .
$$

Вторым слоем для озера Сульфат является вода глубиной до $7 \mathrm{~m}$, для которой $\varepsilon_{2}=81$ и $\rho_{2}=1.1 \Omega \mathrm{m}$. Здесь в диапазоне частот $100 \mathrm{kHz}-10 \mathrm{MHz}$ величина $1 / \varepsilon_{0} \omega \rho_{2}=2 \cdot 10^{6}-2000$, что много больше, чем $\varepsilon_{2}=81$. Поэтому для второго слоя

$$
k_{2}^{2}=\frac{i \mu_{0} \omega}{\rho_{2}} .
$$

Здесь магнитная постоянная вакуума $\mu_{0}=1 / \varepsilon_{0} c^{2}$. Скинслой [1-3] в диапазоне частот $100 \mathrm{kHz}-10 \mathrm{MHz}$ для 
рассматриваемой воды

$$
\sqrt{\frac{2 \rho_{2}}{\mu_{0} \omega}}=1.5-0.15 \mathrm{~m} \text {. }
$$

Эта величина существенно меньше глубины озера, поэтому второй слой можно считать неограниченным.

\section{Компоненты электромагнитного поля}

Приводимые ниже вычисления в предлагаемом разделе во многом повторяют аналогичные вычисления в работе [5]. Здесь только явно учтена двухслойность подстилающей среды.

Выбираем цилиндрическую систему координат, такую, что радиальная координата $r$ лежит на поверхности раздела свободное пространство-среда, ось $z$ направлена по нормали от поверхности раздела в свободное пространство. Распространение поля происходит вдоль радиальной координаты $r$, вектор магнитного поля Н вблизи земной поверхности имеет только одну ненулевую компоненту $H_{y}(r, z)$ :

$$
\mathbf{H}=\left(0, H_{y}(r, z), 0\right) .
$$

Компоненты электрического поля будут находиться из следующих выражений:

$$
E_{r}=-\frac{i \mu_{0} \omega}{k^{2}} \frac{\partial}{\partial z} H_{y}, \quad E_{z}=-\frac{i \mu_{0} \omega}{k^{2}} \frac{1}{r} \frac{\partial}{\partial r}\left(r, H_{y}\right) .
$$

Временная зависимость дается множителем $\exp (-i, \omega, t)$, он одинаков для всех компонент полей и обычно не выписывается. В цилиндрической системе координат при полной угловой симметрии волновое уравнение (уравнение Гельмгольца) для магнитного поля принимает следующий вид:

$$
\frac{1}{r} \frac{\partial}{\partial r}\left(r \frac{\partial H_{y}}{\partial r}\right)+\frac{\partial^{2} H_{y}}{\partial z^{2}}+k^{2} H_{y}=0 .
$$

Решение уравнения (3) в каждой из сред в волновой зоне будет следующим:

$$
\begin{gathered}
H_{0 y}(r, z)=A \frac{1}{\sqrt{r}} \exp \left(i \lambda r-i \sqrt{k_{0}^{2}-\lambda^{2} z}\right), \\
H_{1 y}(r, z)=B \frac{1}{\sqrt{r}} \exp (i \lambda r) \\
\times\left(\exp \left(-i \sqrt{k_{1}^{2}-\lambda^{2} z}\right)+V \exp \left(i \sqrt{k_{1}^{2}-\lambda^{2}} z\right)\right), \\
H_{2 y}(r, z)=D \frac{1}{\sqrt{r}} \exp \left(i \lambda r-i \sqrt{k_{2}^{2}-\lambda^{2}} z\right) .
\end{gathered}
$$

Здесь $\lambda$ - параметр разделения переменных в уравнении (3). Для квадратов волновых чисел, как выше установили, имеем

$$
k_{0}^{2}=\frac{\omega^{2}}{c^{2}}, \quad k_{1}^{2}=\frac{\omega^{2}}{c^{2}}\left(\varepsilon_{1}+\frac{i}{\varepsilon_{0} \omega \rho_{1}}\right), \quad k_{2}^{2}=\frac{i \mu_{0} \omega}{\rho_{2}} .
$$

В диапазоне частот $100 \mathrm{kHz}-10 \mathrm{MHz}$ величины $\left|k_{2}\right|=$ $=3 \cdot 10^{3}-3 \cdot 10^{4}$ и $\left|k_{1}\right|=3 \cdot 10^{-3}-3 \cdot 10^{-1}$, т. е. $k_{2}^{2} \gg k_{1}^{2}$. Ниже установим, что $\lambda \approx \omega / c$ и в рассматриваемом диапазоне частот $k_{2}^{2} \gg \lambda^{2}$.

Из (2) находим компоненты электрического поля в свободном пространстве (в волновой зоне дифференцирование множителя $1 / \sqrt{r}$ не производится)

$$
\begin{gathered}
E_{0 z}(r, z)=-\frac{\mu_{0} \omega \lambda}{k_{0}^{2}} A \frac{1}{\sqrt{r}} \exp \left(i \lambda r-i \sqrt{k_{0}^{2}-\lambda^{2} z}\right), \\
E_{0 r}(r, z)=-\mu_{0} \omega \frac{\sqrt{k_{0}^{2}-\lambda^{2}}}{k_{0}^{2}} A \frac{1}{\sqrt{r}} \exp \left(i \lambda r-i \sqrt{k_{0}^{2}-\lambda^{2}} z\right) .
\end{gathered}
$$

В диэлектрическом первом слое

$$
\begin{aligned}
E_{1 z}(r, z)= & -\frac{\mu_{0} \omega \lambda}{k_{1}^{2}} B \frac{1}{\sqrt{r}} \exp (i \lambda r)\left(\exp \left(-i \sqrt{k_{1}^{2}-\lambda^{2}} z\right)\right. \\
& \left.+V \exp \left(i \sqrt{k_{1}^{2}-\lambda^{2}} z\right)\right) \\
E_{1 r}(r, z)= & -\mu \omega \frac{\sqrt{k_{1}^{2}-\lambda^{2}}}{k_{1}^{2}} B \frac{1}{\sqrt{r}} \exp (i \lambda r) \\
\quad & \left(\exp \left(-i \sqrt{k_{1}^{2}-\lambda^{2} z}\right)-V \exp \left(i \sqrt{k_{1}^{2}-\lambda^{2}} z\right)\right)
\end{aligned}
$$

В проводящей неограниченной вглубь среде

$$
\begin{gathered}
E_{2 z}(r, z)=-\frac{\mu_{0} \omega \lambda}{k_{2}^{2}} D \frac{1}{\sqrt{r}} \exp \left(i \lambda r-i \sqrt{k_{2}^{2}-\lambda^{2}} z\right), \\
E_{2 r}(r, z)=-\mu_{0} \omega \frac{\sqrt{k_{2}^{2}-\lambda^{2}}}{k_{2}^{2}} D \frac{1}{\sqrt{r}} \exp \left(i \lambda r-i \sqrt{k_{2}^{2}-\lambda^{2}} z\right) .
\end{gathered}
$$

Приведенные формулы позволяют определить все характеристики электромагнитного поля в любой из двухслойных сред. Мы ниже найдем основную характеристику подстилающей среды - его импеданс.

\section{Трансцендентное уравнение для параметра разделения}

Граничные условия при $z=0$ имеют следующий вид:

$$
\begin{aligned}
& E_{0 r}(r, z=0)=E_{1 r}(r, z,=0), \\
& H_{0 y}(r, z=0)=H_{1 y}(r, z=0) .
\end{aligned}
$$

Из них находим

$$
\begin{aligned}
A \frac{\sqrt{k_{0}^{2}-\lambda^{2}}}{k_{0}^{2}} & =B \frac{\sqrt{k_{1}^{2}-\lambda^{2}}}{k_{1}^{2}}(1-V), \\
A & =B(1+V) .
\end{aligned}
$$


Разделив их друг на друга, находим коэффициент $V$ :

$$
V=\frac{1-Q_{10}}{1+Q_{10}}, \quad Q_{10}=\frac{k_{1}^{2}}{k_{0}^{2}} \frac{\sqrt{k_{0}^{2}-\lambda^{2}}}{\sqrt{k_{1}^{2}-\lambda^{2}}} .
$$

При $z=-h$ граничные условия имеют вид

$$
\begin{aligned}
& E_{2 r}(r, z=-h)=E_{1 r}(r, z=-h), \\
& E_{2 y}(r, z=-h)=H_{1 y}(r, z=-h) .
\end{aligned}
$$

Отсюда

$$
\begin{gathered}
\frac{\sqrt{k_{1}^{2}-\lambda^{2}}}{k_{1}^{2}} B\left(\exp \left(i \sqrt{k_{1}^{2}-\lambda^{2}} h\right)-V \exp \left(-i \sqrt{k_{1}^{2}-\lambda^{2}} h\right)\right) \\
=\frac{\sqrt{k_{2}^{2}-\lambda^{2}}}{k_{2}^{2}} D \exp \left(i \sqrt{k_{2}^{2}-\lambda^{2}} h\right), \\
B\left(\exp \left(i \sqrt{k_{1}^{2}-\lambda^{2}} h\right)+V \exp \left(-i \sqrt{k_{1}^{2}-\lambda^{2}} h\right)\right) \\
=D \exp \left(i \sqrt{k_{2}^{2}-\lambda^{2}} h\right) .
\end{gathered}
$$

Разделив их друг на друга, для коэффициента $V$ находим другое выражение

$$
\begin{gathered}
V=\exp \left(2 i \sqrt{k_{1}^{2}-\lambda^{2}} h\right) \frac{1-Q_{12}}{1+Q_{12}}, \\
Q_{12}=\frac{k_{1}^{2}}{k_{2}^{2}} \frac{\sqrt{k_{2}^{2}-\lambda^{2}}}{k_{1}^{2}-\lambda^{2}} .
\end{gathered}
$$

Постоянные $A, B$ и $D$ ниже никак не сказываются при вычислении импеданса, и мы их исключили. Приравнивая соотношения (14) и (15), находим уравнение для определения параметра $\lambda$ :

$$
\frac{1-Q_{10}}{1+Q_{10}}=\exp \left(2 i \sqrt{k_{1}^{2}-\lambda^{2}} h\right) \frac{1-Q_{12}}{1+Q_{12}} \text {. }
$$

Это уравнение является трансцендентным и решается только численно. Для этого написана программа „Импеданс“ [6]. Однако рассматриваемая двухслойная среда имеет большой контраст электрофизических свойств слоев льда и воды, что позволяет выделить величины разного порядка, и при этом становится возможным аналитическое решение уравнения (16). Ниже найдем решение уравнения (16) в двух последовательных приближениях. Для получения первого приближения достаточно пренебречь квадратом импеданса. Во втором приближении для импеданса получим выражение, которое позволяет явно учесть небольшую толщину первого слоя.

\section{Импеданс}

Импеданс $\delta$ определяется следующим выражением [1-3]:

$$
\delta=-\frac{1}{\mu_{0} c}\left(\frac{E_{r}}{H_{y}}\right)_{z=0} .
$$

Знак минус связан с выбором ориентации оси $z$. Подставляя в определение (17) выражения (4) и (9), находим $\delta=\sqrt{1-\lambda^{2} / k_{0}^{2}}$ или

$$
\lambda=k_{0} \sqrt{1-\delta^{2}}
$$

Этим самым параметр разделения волнового уравнения (3) выразили через поверхностный импеданс. По своему определению квадрат модуля импеданса удовлетворяет неравенству $|\delta|^{2} \ll 1$. Тогда из (18) следует $\lambda \approx k_{0}$, что указывалось выше.

\section{Импеданс в первом приближении}

Используя неравенство $\left|\delta^{2}\right| \ll 1$ и пренебрегая в трансцендентном уравнении (16) величиной $\delta^{2}$, сначала получаем

$$
\frac{1-Q_{10}}{1+Q_{10}}=W \frac{1-Q_{12}}{1+Q_{12}} \equiv K .
$$

Здесь ввели экспоненту

$$
W=\exp \left(2 i \frac{\omega h}{c} \sqrt{\varepsilon_{1}-1+\frac{i}{\varepsilon_{0} \omega \rho_{1}}}\right) .
$$

Величины $Q_{10}$ и $Q_{12}$ даются выражениями (14) и (15), причем $\delta^{2}$ отброшено. Далее, используя выражение (7) для $k_{1}^{2}$, из (19) окончательно находим аналитическое выражение для импеданса

$$
\delta=\frac{\sqrt{\varepsilon_{1}-1+i / \varepsilon_{0} \omega \rho_{1}}}{\varepsilon_{1}+i / \varepsilon_{0} \omega \rho_{1}} \frac{1-K}{1+K} .
$$

Полученное выражение (21) еще можно упростить. Для этого надо учесть, что $\lambda \approx k_{0}$. Кроме того, для проводящей подстилающей среды в широком частотном диапазоне $k_{2}^{2} \gg k_{0}^{2}$ и $k_{2}^{2} \gg \lambda^{2}$. Эти неравенства позволяют для введенных величин $Q_{10}$ и $Q_{12}$ приближенно записать:

$$
Q_{10}=\Lambda \delta \ll 1, \quad Q_{12}=\Lambda \sqrt{i \varepsilon_{0} \omega \rho_{2}} \ll 1,
$$

где

$$
\Lambda=\frac{\varepsilon_{1}+i / \varepsilon_{0} \omega \rho_{1}}{\sqrt{\varepsilon_{1}-1+i / \varepsilon_{0} \omega \rho_{1}}} .
$$

Тогда из (16) сначала приближенно имеем

$$
1-2 \Lambda \delta=W\left(1-2 \Lambda \sqrt{-i \varepsilon_{0} \omega \rho_{2}}\right) .
$$

Отсюда находим импеданс

$$
\delta=W \sqrt{-i \varepsilon_{0} \omega \rho_{2}}+\frac{1-W}{2 \Lambda} .
$$

Это выражение для импеданса верно в предположении, что $|\delta| \ll 1$ и $\sqrt{\varepsilon_{0} \omega \rho_{2}} \ll 1$. 


\section{Предельный переход}

В отсутствие льда имеем однородную проводящую среду с импедансом $\delta_{2}=\sqrt{-i \varepsilon_{0} \omega \rho_{2}}$ [1]. Здесь учтено, как указывалось выше, что в диапазоне частот $100 \mathrm{kHz}-10 \mathrm{MHz}$ для воды $1 / \varepsilon_{0} \omega \rho_{2} \gg \varepsilon_{2}$. В рассматриваемом нами случае отсутствие первой среды означает либо устремление толщины льда $h$ к 0 , либо диэлектрической проницаемости льда $\varepsilon_{1}$ к 1 . В первом случае, устремляя $h \rightarrow 0$, находим экспоненту $W \rightarrow 1$. Тогда из (23) сразу находим, что $\delta \rightarrow \sqrt{-i \varepsilon_{0} \omega \rho_{2}}$, как и должно быть. Во втором случае предельный переход $\varepsilon_{1} \rightarrow 1$ требует более подробного рассмотрения. Чтобы упростить рассмотрение, предположим, что величина $\sqrt{\varepsilon_{0} \omega \rho_{1}}$ заметно меньше комбинации $\sqrt{\varepsilon_{1}-1} / \varepsilon_{1}$. Тогда экспоненту (20) можно приближенно записать как

$$
\begin{gathered}
W=\exp \left(2 i \sqrt{k_{1}^{2}-\lambda^{2}} h\right) \approx \exp (2 i \Psi) \\
=\cos (2 \Psi)+i \sin (2 \Psi),
\end{gathered}
$$

где

$$
\Psi=\sqrt{\varepsilon_{1}-1} \frac{\omega h}{c} .
$$

Теперь из (16) приближенно имеем

$$
1-2 Q_{10}=(\cos 2 \Psi+i \sin 2 \Psi)\left(1-2 Q_{12}\right) .
$$

Причем здесь

$$
Q_{10}=\frac{\varepsilon_{1}}{\sqrt{\varepsilon_{1}-1}} \delta \ll 1, \quad Q_{12}=\frac{\varepsilon_{1}}{\sqrt{\varepsilon_{1}-1}} \sqrt{-i \varepsilon_{0} \omega \rho_{2}} \ll 1 .
$$

В итоге из (25) находим

$$
\begin{aligned}
\delta= & \sqrt{\frac{\varepsilon_{0} \omega \rho_{2}}{2}}(\cos 2 \Psi+\sin 2 \Psi)+\frac{\sqrt{\varepsilon_{1}-1}}{2 \varepsilon_{1}}(1-\cos 2 \Psi) \\
& -i\left[\sqrt{\frac{\varepsilon_{0} \omega \rho_{2}}{2}}(\cos 2 \Psi-\sin 2 \Psi)+\frac{\sqrt{\varepsilon_{1}-1}}{2 \varepsilon_{1}} \sin 2 \Psi\right] .
\end{aligned}
$$

Здесь, устремляя $\varepsilon_{1} \rightarrow 1$, сначала находим $\Psi \rightarrow 0$, а затем уже $\delta=\sqrt{-i \varepsilon_{0} \omega \rho_{2}}$. И здесь находим правильное предельное выражение.

\section{Импеданс во втором приближении}

Выражение (23) допускает дальнейшее упрощение. Как показывают проведенные выше оценки, для первого слоя величины $1 / \varepsilon_{0} \omega \rho_{1}$ и $\varepsilon_{1}$ одного порядка. Поэтому необходимо пользоваться выражением (7) для квадрата волнового числа $k_{1}^{2}$ первой среды. Если предположить, что аргумент в экспоненте (20) мал, то из (23) получаем во втором приближении следующее выражение для импеданса:

$$
\delta=\sqrt{-i \varepsilon_{0} \omega \rho_{2}}-i \frac{\omega h}{c}\left(1-\frac{1}{\varepsilon_{1}+i / \varepsilon_{0} \omega \rho_{1}}\right) .
$$

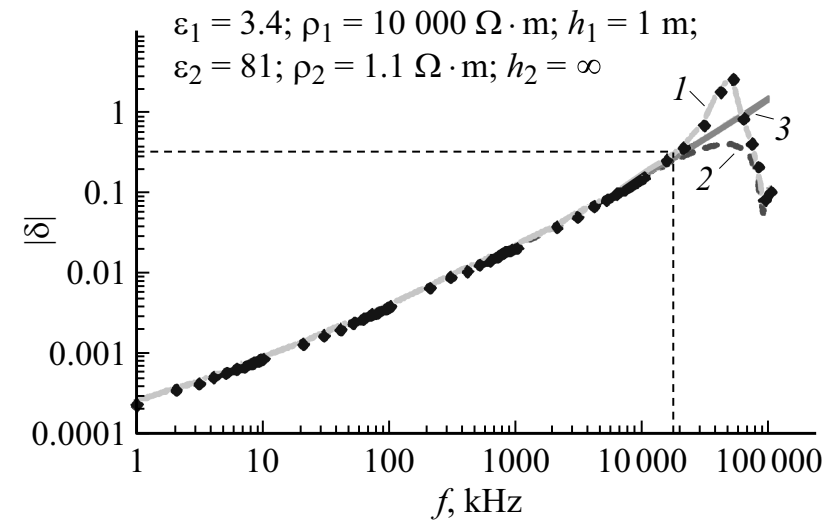

Рис. 1. Зависимость модуля импеданса от частоты для структуры „лед на соленой воде“. Сплошная линия с квадратиками 1 - вычисленные по программе „Импеданс“ [6], штриховая линия 2 - вычисленные по формуле (21), сплошная линия 3 - вычисленные по формуле (26). Прямоугольная область, ограниченная штриховыми прямыми, - область выполнения импедансных граничных условий Леонтовича.

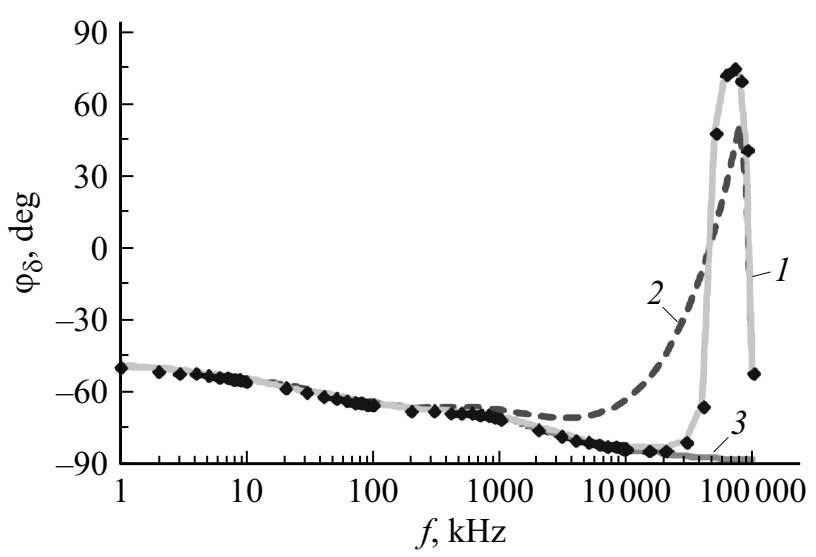

Рис. 2. Зависимость фазы импеданса от частоты. Обозначения те же, что и для рис. 1.

Отсюда следуют два важных вывода. Если первая среда в некотором частотном диапазоне является проводящей (например, очень грязный лед), т.е. выполняется неравенство

$$
1 / \varepsilon_{0} \omega \rho_{1} \gg \varepsilon_{1},
$$

то выражением $\left(\varepsilon_{1}+1 / \varepsilon_{0} \omega \rho_{1}\right)^{-1}$ по сравнению с 1 можно пренебречь. И если обозначить $\delta_{2}=\sqrt{-i \varepsilon_{0} \omega \rho_{2}}$, то из (26) получаем

$$
\delta=\delta_{2}-i \frac{\omega h}{c} .
$$

Этим самым находим поправку на импеданс при наличии тонкого проводящего слоя [2,7]. Заметим также, что для импеданса $\delta_{2}$ можно получить точное выражение:

$$
\delta_{2}=k_{0} \sqrt{k_{2}^{2}-k_{0}^{2}} / k_{2}^{2} .
$$


В противоположном предельном случае, когда первый слой является диэлектриком с диэлектрической проницаемостью $\varepsilon_{1}$, т.е. для него в некотором частотном диапазоне выполняется неравенство

$$
1 / \varepsilon_{0} \omega \rho_{1} \ll \varepsilon_{1},
$$

то из (26) следует

$$
\delta=\delta_{2}-i \frac{\varepsilon_{1}-1}{\varepsilon_{1}} \frac{\omega h}{c} .
$$

Этим самым находим поправку на импеданс при наличии тонкого диэлектрического слоя.

\section{Графическое представление импеданса}

Важным представлением земной поверхности является его моделирование многослойной средой [8]. Для вычисления поверхностного импеданса написана программа „Импеданс“ [6]. Эта программа позволяет численным способом также решать трансцендентное уравнение (16). Результаты вычислений для озера Сульфат, для которого известны $h=1 \mathrm{~m}, \varepsilon_{1}=3.2, \rho_{1}=10^{4} \Omega \mathrm{m}$ и $\rho_{2}=1.1 \Omega \mathrm{m}, \varepsilon_{2}=81$, представлены для модуля импеданса на рис. 1 и для фазы импеданса на рис. 2. Там же показаны результаты, вычисленные по формулам (21) и (26). Анализ рисунков показывает, что для модуля импеданса $|\delta|$ результаты совпадают вплоть до $20 \mathrm{MHz}$. Для фазы импеданса $\varphi_{\delta}$ вычисление по формуле (21) несколько хуже, совпадение наблюдается только до $3 \mathrm{MHz}$. Вычисление по формуле (26) для фазы импеданса $\varphi_{\delta}$ необходимо распространить до $20 \mathrm{MHz}$. В любом случае фаза импеданса $\varphi_{\delta}$ лежит в пределах $-45.1^{0}--89.9^{\circ}$. Это указывает, что рассматриваемая поверхность является сильно индуктивной и может служить для целей дальней радиосвязи.

Из рис. 1 и 2 следует, что до частот $20 \mathrm{MHz}$ выполняется условие $|\delta|^{2} \ll 1$, при этом результаты вычислений для модуля и фазы импеданса практически совпадают с численным решением и по формуле (26). Поэтому при расчетах импеданса в большинстве случаев достаточно пользоваться формулой (26). Кроме того, укажем, что выполнение условия $|\delta|^{2} \ll 1$ является выполнением импедансного граничного условия Леонтовича $[5,9]$. В работе [9] отмечено, что сравнение точного решения уравнений Максвелла с приближенными возможно только как раз при выполнении условия $|\delta|^{2} \ll 1$.

\section{Заключение}

Рассмотрена двухслойная среда „свободное пространство-диэлектрический слой конечной толщины-проводящая неограниченная вглубь среда“. Такая двухслойная среда в определенном частотном диапазоне радиоволн позволила выделить малые величины и получить конечное аналитическое выражение (26) для приведенного поверхностного импеданса. Это выражение для импеданса сравнили с численным расчетом для многослойной среды, которое показало удовлетворительное согласие.

Работа выполнена в рамках госбюджетной темы „Распространение радиоволн в неоднородных импедансных каналах“.

\section{Список литературы}

[1] Балханов В.К., Башкуев Ю.Б. Основы теории метода поверхностного импеданса. Улан-Уде. Изд-во Бурятского научного центра СО РАН, 2005.

[2] Макаров Г.И., Новиков В.В., Рыбачек С.Т. Распространение электромагнитных волн над земной поверхностью. М.: Наука, 1991.

[3] Wait J.R. Electromagnetic waves in stratified media. Oxford, NY: Pergamon Press, 1996.

[4] Башкуев Ю.Б., Хаптанов В.Б., Дембелов М.Г. // Письма в ЖТФ. 2010. Т. 36. Вып. 3. С. 88-95.

[5] Балханов В.К., Башкуев Ю.Б. // ЖТФ. 2017. Т. 87. Вып. 4. C. $599-603$.

[6] Ангархаева Л.Х. Пакет программ „Импеданс“ для решения задач радиоимпедансного зондирования: свидетельство об официальной регистрации программы для ЭВМ № 2002610893. РФ / заявл. 11.04.2002; зарегистр. 06.06.2002.

[7] Башкуев Ю.Б., Нагуслаева И.Б., Хаптанов В.Б., Дембелов М.Г. // ЖТФ. 2016. Т. 86. Вып. 2. С. 153-155.

[8] Балханов В.К., Башкуев Ю.Б., Ангархаева Л.Х. и др. // ЖТФ. 2014. Т. 84. Вып. 11. С. 101-105.

[9] Бердник С.Л., Пенкин Д.Ю., Катрич В.А. и др. // Радиофизика и радиоастрономия. 2014. Т. 19. № 1. С. 57-80. 